\title{
Distal 7q11.23 microdeletion syndrome
}

INSERM

\section{Source}

INSERM. (1999). Orphanet: an online rare disease and orphan drug data base. Distal 7q11.23 microdeletion syndrome. ORPHA:254351

Distal 7q11.23 microdeletion syndrome is a rare chromosomal anomaly characterized by epilepsy, neurodevelopmental disorder variably including developmental delays and intellectual disabilities of variable severity, learning disability and neurobehavioral abnormalities (autism spectrum disorder, hyperactivity, impulsivity, agg ression, selfabusive behaviors, depression). 